\title{
Coulomb scattering in a 2D interacting electron gas and production of EPR pairs
}

\author{
D. S. Saraga ${ }^{1}$, B. L. Altshuler ${ }^{2,3}$, Daniel Loss ${ }^{1}$, and R. M. Westervelt ${ }^{4}$ \\ ${ }^{1}$ Department of Physics and Astronomy, University of Basel, Klingelbergstrasse 82, CH-4056 Basel, Switzerland \\ ${ }^{2}$ Physics Department, Princeton University, Princeton, New Jersey 08544 \\ ${ }^{3} \mathrm{NEC}$ Research Institute, 4 Independence Way, Princeton, New Jersey 08540 and \\ ${ }^{4}$ Division of Engineering and Applied Sciences, Harvard University, Cambridge, Massachusetts 02138
}

(Dated: 11th November 2018)

\begin{abstract}
We propose a setup to generate non-local spin-EPR pairs via pair collisions in a $2 \mathrm{D}$ interacting electron gas, based on constructive two-particle interference in the spin singlet channel at the $\pi / 2$ scattering angle. We calculate the scattering amplitude via the Bethe-Salpeter equation in the ladder approximation and small $r_{s}$ limit, and find that the Fermi sea leads to a substantial renormalization of the bare scattering process. From the scattering length we estimate the current of spin-entangled electrons and show that it is within experimental reach.

PACS numbers : 73.23.-b, 71.10.Ca, 3.67.Mn,
\end{abstract}

In recent years, the spin degree of freedom of electrons has become of central interest in semiconductor research 1]. This is particularly so for spin-based quantum information processing, where the basic resources are EPR pairs such as non-local spin singlets formed by two electrons that are spatially separated 2]. A number of recent publications have described ways to produce such spincorrelated two-electron states, as well as orbital entanglement $3,4,5,6]$. Here, we propose a new concept based on a two-particle interference mechanism that is wellknown from elementary scattering theory [7]: the crosssection for two electrons in vacuum, given in terms of the scattering amplitude $f$ by $\lambda_{S / T}(\theta)=|f(\theta) \pm f(\pi-\theta)|^{2}$, favors singlet $(+)$ over triplet $(-)$ states in the outgoing channel around the scattering angle $\theta=\pi / 2$. Thus, in principle, two-particle scattering processes can be used to generate EPR pairs. However, in the context of solid state systems, the question immediately arises whether this scattering effect remains operational, and moreover observable, in the presence of a Fermi sea consisting of many interacting electrons such as a typical two-dimensional electron gas (2DEG) formed in GaAs heterostructures. In this Letter, we will show that within Fermi liquid theory the answer is affirmative.

We focus on 2DEGs since these systems are promising candidates for the observation of such effects. Indeed, recent experiments [8] have demonstrated that in a 2DEG the flow of electrons as well as scattering off impurities can be controlled and monitored via AFM technology. Thus, we believe that a setup as shown in Fig. 1(a) is experimentally realizable and should allow the observation of the angular and density dependence of the scattering cross section. Once the EPR pairs are created their singlet character can be tested 9] by a noise measurement in a beam splitter configuration [2], or by tests of Bell inequalities [10].

We note that the experimental observation of this type of entanglement would provide support for the applicability of Fermi liquid theory to 2D systems, which has been questioned by Anderson [11]. Indeed, one
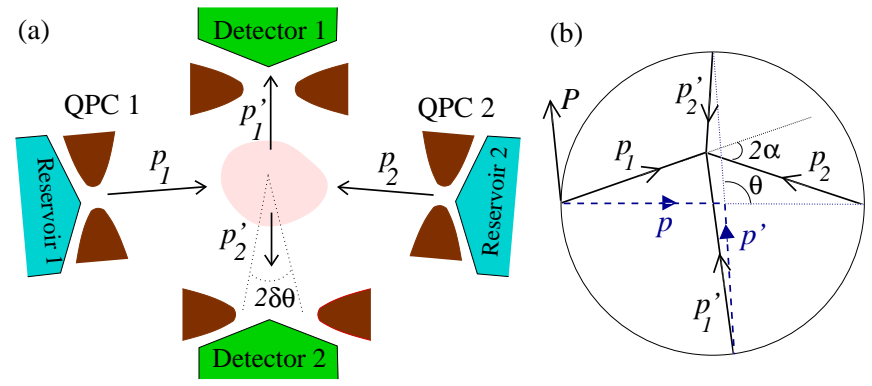

Figure 1: (a) Proposed setup: two quantum point contacts (QPC) filter electrons from two reservoirs with initial momenta $\mathbf{p}_{1} \simeq-\mathbf{p}_{2}$. The two detectors (with an aperture angle $2 \delta \theta$ ) are placed such that only electrons which collide (shaded area) at a scattering angle around $\pi / 2$ are registered. Because of interference, the scattering amplitude $f$ vanishes at $\pi / 2$ for the spin-triplet states, allowing only the spin-entangled singlets to be collected: one electron of the singlet state in detector 1 and its partner in detector 2 . The scattering crosssection and the electron flux could be measured via an AFM tip [8]. (b) Scattering parameters: $\mathbf{P}=\mathbf{p}_{1}+\mathbf{p}_{2}=\mathbf{p}_{1}^{\prime}+\mathbf{p}_{2}^{\prime}$ is the total momentum, $\mathbf{p}=\left(\mathbf{p}_{1}-\mathbf{p}_{2}\right) / 2$ and $\mathbf{p}^{\prime}=\left(\mathbf{p}_{1}^{\prime}-\mathbf{p}_{2}^{\prime}\right) / 2$ are the relative momenta, and $\theta=\angle\left(\mathbf{p}, \mathbf{p}^{\prime}\right)$ is the scattering angle between them. The initial $\left(\mathbf{p}_{1}, \mathbf{p}_{2}\right)$ and final $\left(\mathbf{p}_{1}^{\prime}, \mathbf{p}_{2}^{\prime}\right)$ momenta are connected by a circle of radius $p=p^{\prime}$ due to energy and momentum conservation.

can hardly imagine singlet pairs of particles that are separated by mesoscopic distances without well-defined fermionic quasi-particles.

For electrons incident from unpolarized sources, we expect the ratio of singlets $|S\rangle$ to triplets $\left|T_{i}\right\rangle, i=0, \pm$ to be $1: 3$, this mixed state being described by $1 / 4|S\rangle\langle S|+$ $1 / 4 \sum_{i=0, \pm}\left|T_{i}\right\rangle\left\langle T_{i}\right|$. Our goal is to calculate the ratio, of scattered triplets to scattered singlets, for an aperture angle $2 \delta \theta$ of the detectors around $\theta=\pi / 2$. For small $\delta \theta$ this ratio is $R \simeq \delta \theta^{2}\left|f^{\prime} / f\right|_{\theta=\pi / 2}^{2}$, where $f$ is the manybody scattering amplitude and $f^{\prime}$ its angular derivative. Solving the Bethe-Salpeter equation in the small $r_{s}$ limit we find that $\left|f^{\prime} / f\right|^{2} \sim 1$ at $\theta=\pi / 2$. This means that for small $\delta \theta$ the scattering process produces dominantly singlets in the direction of the detectors 1 and 2 ; see 
Fig. 1(a). As an experimental check, this ratio can be increased by reducing the amount of singlets in the incoming channels, which in turn can be achieved e.g. by using spin polarized electron sources or devices that act as spin filters such as a quantum dot [12] or a quantum point contact [13].

Setup. We begin with the description of the setup, shown in Fig. 1(a). Electrons escaping from thermal reservoirs with momenta $\mathbf{p}_{1} \simeq-\mathbf{p}_{2}$ are filtered by two quantum point contacts (QPC) and injected into a $2 \mathrm{DEG}$ where they scatter off each other. To collect after the collision only the entangled singlets (EPR pairs), we place two detectors such that only collisions of electrons with final momenta $\mathbf{p}_{1}^{\prime}, \mathbf{p}_{2}^{\prime}$ and with scattering angle $\theta \in[\pi / 2-\delta \theta, \pi / 2+\delta \theta]$ are registered. Below we estimate the expected singlet flux (current).

As seen from Fig. I $(b)$, the energies of both incident electrons need to be known, in general, to determine the scattering angle $\theta \simeq \angle\left(\mathbf{p}^{\prime}, \mathbf{p}\right)$. However, for the special case with opposite momenta $\mathbf{p}_{2} \simeq-\mathbf{p}_{1}, \theta$ is easily determined by $\theta \simeq \angle\left(\mathbf{p}_{1}^{\prime}, \mathbf{p}_{1}\right)$. Moreover, the energies are then individually conserved, $p_{1} \simeq p_{2} \simeq p_{1}^{\prime} \simeq p_{2}^{\prime}\left(p_{i}=\left|\mathbf{p}_{i}\right|\right)$, which ensures that the outgoing scattering states are unoccupied $\left(p_{1,2}^{\prime}>k_{F}\right)$. Finally, to have well-defined quasiparticle states with long lifetimes, we assume the electrons to be injected with small excitation energies $\xi_{i}=\hbar^{2} p_{i}^{2} / 2 m-E_{F} \ll E_{F}$, where $E_{F}=\hbar^{2} k_{F}^{2} / 2 m$ is the Fermi energy and $m$ the effective mass.

$S$ cattering $t$-matrix. Let us evaluate the scattering $t$ matrix for two electrons in the presence of the Fermi sea. The condition $\mathbf{p}_{2}=-\mathbf{p}_{1}$ defines the Cooper channel, and thus we can follow the work by Kohn and Luttinger on interaction-induced superconductivity in a 3D Fermi liquid [14]. In contrast to Ref. 14], we consider here a $2 \mathrm{D}$ system where the screened Coulomb potential is nonanalytic. Moreover, we need the complete angular dependence of the scattering amplitude - rather than only the asymptotics of its Legendre or Fourier coefficients. The $t$-matrix can be obtained from the (direct) vertex $\Gamma$ governed by the Bethe-Salpeter equation 14, 15]

$$
\begin{aligned}
& \Gamma\left(\tilde{p}^{\prime}, \tilde{p} ; \tilde{P}\right)=\Lambda\left(\tilde{p}^{\prime}, \tilde{p} ; \tilde{P}\right) \\
& +\frac{i}{\hbar(2 \pi)^{3}} \int d \tilde{k} \Lambda(\tilde{k}, \tilde{p} ; \tilde{P}) G\left(\tilde{k}_{1}\right) G\left(\tilde{k}_{2}\right) \Gamma\left(\tilde{p}^{\prime}, \tilde{k} ; \tilde{P}\right),
\end{aligned}
$$

where $G$ is the single-particle Green function and $\Lambda$ the irreducible vertex (we suppress the spin indices as spin is conserved). The relative and total 4-momenta are denoted by $\tilde{p}=\left(\tilde{p}_{1}-\tilde{p}_{2}\right) / 2$, and $\tilde{P}=\tilde{p}_{1}+\tilde{p}_{2}$, where $\tilde{p}_{i}=\left(\mathbf{p}_{i}, \omega_{i}\right)$ with the frequencies $\omega_{i}$. The full vertex for singlet/triplets contains the direct and exchange parts, i.e. $\Gamma\left(\tilde{p}^{\prime}, \tilde{p} ; \tilde{P}\right) \pm \Gamma\left(-\tilde{p}^{\prime}, \tilde{p} ; \tilde{P}\right)$. From the vertex we obtain the $t$-matrix: $t=\Gamma\left(\omega_{1}+\omega_{2} \rightarrow \xi_{1}+\xi_{2}\right)$ [15], and from it the (unsymmetrized) scattering length $\lambda=|f|^{2}$ via the $2 \mathrm{D}$ scattering amplitude $f=-t m / \hbar^{2} \sqrt{2 \pi p}$ [16]. The scattering lengths for singlet/triplets are then given by $\lambda_{S / T}(\theta)=|f(\theta) \pm f(\theta-\pi)|^{2}$.
The bare Coulomb interaction in $2 \mathrm{D}$ is given by $V_{C}(\mathbf{q})=2 \pi e_{0}^{2} / q$ with $e_{0}^{2}=e^{2} / 4 \pi \epsilon_{0} \epsilon_{r}$ and the dielectric constant $\epsilon_{r}$. In a first stage, we use the RPA approximation to account for screening by the Fermi sea 15]; this yields a renormalized $G$ and a screened interaction $V(\tilde{q})=V_{C}(q) /\left[1-V_{C}(q) \chi^{0}(\tilde{q})\right]$ given in terms of the bubble susceptibility diagram $\chi^{0}$ with $\tilde{q}=\left(\mathbf{q}, \omega_{q}\right)=\tilde{p}^{\prime}-\tilde{p}$ the momentum transfer. We can consider [17] the usual static Thomas-Fermi screening $V(q)=e_{0}^{2} /\left(q+k_{s}\right)$, with the screening momentum $k_{s}=2 m e_{0}^{2} / \hbar^{2}$ and $r_{s}=k_{s} / k_{F} \sqrt{2}$. Note that RPA requires a high density, $r_{s} \ll 1$.

In addition to the single interaction line $V(q)$, the irreducible vertex $\Lambda$ contains, in lowest order in $V$, two diagrams: the crossed diagram and the "wave function modification" shown in Fig. 2(c) and (d) of Ref. 14]. It is easy to see that these are smaller than $V$ by a factor $r_{s}$ [18]. Altogether, this justifies the ladder approximation, which consists in keeping only the single interaction line in $\Lambda \simeq V$. Note that the ladder approximation requires $\lambda k_{F} \ll 1[15]$. This condition is consistent with RPA: e.g., in the Born approximation $t \simeq V_{C} \Rightarrow$ $\lambda k_{F} \sim\left[V_{C}\left(k_{F}\right) m\right]^{2} / 2 \pi \hbar^{4} \sim\left(k_{s} / k_{F}\right)^{2}$. Finally, we can approximate $G$ by the free propagator $G_{0}$ 14]. We start with the zero temperature $T=0$ case, and discuss finite $T$ effects later.

Before solving Eq.(1) to all orders, we first consider its second-order iteration $\Gamma^{(2)}\left(\mathbf{p}^{\prime}, \mathbf{p} ; \tilde{P}\right)=V\left(\mathbf{p}^{\prime}-\mathbf{p}\right)+$ $i / \hbar(2 \pi)^{3} \int d \tilde{k} V(\mathbf{k}-\mathbf{p}) G\left(\tilde{k}_{1}\right) G\left(\tilde{k}_{2}\right) V\left(\mathbf{p}^{\prime}-\mathbf{k}\right)$. The $\omega-$ integration of the Green functions yields [15]

$$
\begin{aligned}
& \frac{i}{2 \pi \hbar} \int d \omega_{k} G_{0}\left(\mathbf{k}_{1}, \frac{\Omega}{2}+\omega_{k}\right) G_{0}\left(\mathbf{k}_{2}, \frac{\Omega}{2}-\omega_{k}\right) \\
= & \frac{N\left(\mathbf{k}_{1}, \mathbf{k}_{2}\right)}{\hbar \Omega-\xi_{k_{1}}-\xi_{k_{2}}+2 i \eta N\left(\mathbf{k}_{1}, \mathbf{k}_{2}\right)}=: D\left(\mathbf{k}_{1}, \mathbf{k}_{2}\right),
\end{aligned}
$$

with $N\left(\mathbf{k}_{1}, \mathbf{k}_{2}\right)=1-n\left(k_{1}\right)-n\left(k_{2}\right), n(k)=\Theta\left(-\xi_{k}\right)$, and the $\tilde{P}$-frequency is $\hbar \Omega \rightarrow \xi_{1}+\xi_{2}$ to obtain the $t$-matrix. We now take $\mathbf{p}=\mathbf{p}_{1}=-\mathbf{p}_{2}, \mathbf{k}=\mathbf{k}_{1}=-\mathbf{k}_{2}$ so that $q=$ $\left|\mathbf{p}^{\prime}-\mathbf{p}\right|=2 k_{F}|\sin \theta / 2|$. This yields a single sharp edge in the numerator when $\xi_{k}=0$, which coincides with the zero of the denominator at $\xi_{k}=\xi$ for incident electrons with vanishing excitation energies $\xi=\xi_{1,2} \rightarrow 0$. Thus, the main contribution to the energy integration comes from virtual states at the Fermi surface, i.e. $\xi_{k} \simeq 0$. We set $k=k_{F}$ in $V$, and integrate only on $D(k)=D(\mathbf{k}, \mathbf{k})$. The dominant contribution comes from the vicinity of the Fermi sphere $k=k_{F}$. Introducing the notation $\nu:=$ $(1 / 2 \pi) \int_{0}^{\infty} d k k D(k)$, we obtain

$$
\nu \simeq \frac{m}{2 \pi \hbar^{2}} \log \frac{\xi}{E_{F}} .
$$

For finite temperatures with $\xi \ll k_{B} T \ll E_{F}$, the occupation function $n(k)=\left(1+e^{\xi_{k} / k_{B} T}\right)^{-1}$ cuts the log divergence and $\xi$ is replaced by $k_{B} T$ in Eq. (3). This logarithmic divergence reveals a $2 \mathrm{D}$ Cooper singularity (see below) very much like in 3D [15]. Next, we 
examine the situation slightly away from the Cooper channel, i.e. for non-vanishing total momentum. Indeed, for experimental reasons it is preferable to have a small but finite angle $2 \alpha=L\left(\mathbf{p}_{1},-\mathbf{p}_{2}\right)$ between the incident particles to avoid flux misalignment with no collision at all. In this case, at $T=0$ and for $p_{1}=p_{2}$, we find that $N(k, \phi) \simeq \Theta\left(k-k_{F}-p \alpha|\sin \phi|\right)-$ $\Theta\left(k_{F}-k-p \alpha|\sin \phi|\right)$ depends on the integration angle $\phi=\angle(\mathbf{k}, \mathbf{p})$ and, as a consequence, we find now $\nu(\phi) \simeq\left(m / 2 \pi \hbar^{2}\right) \log (2 \alpha|\sin \phi|)$ in the limit $p \rightarrow k_{F}$. Thus the cut-off of the log-divergence is determined by $\max \left\{\alpha|\sin \phi|, \xi / E_{F}, k_{B} T / E_{F}\right\}$. However, we recall that both outgoing scattering states must be unoccupied, which at $T=0$ requires $p_{1,2}^{\prime}>k_{F}$. For $\pi / 2$-scattering, $p_{1,2}^{\prime}=p_{1}(\cos \alpha \pm \sin \alpha) \Rightarrow p_{1} \gtrsim k_{F}(1+\alpha)$; see Fig. 1(b). Therefore, $\alpha<\xi / E_{F}$ and the relevant cut-off is still given by $\xi$ or $k_{B} T$.

We repeat this procedure in each order in $V$ and rewrite Eq. (1) as $t(\theta)=v(\theta)+(\nu / 2 \pi) \int \mathrm{d} \phi v(\phi) t(\theta-\phi)$, with $v(\phi)=2 \pi e^{2} /\left(2 k_{F}|\sin \phi / 2|+k_{s}\right)$ the $2 \mathrm{D}$ Coulomb potential $\mathrm{V}$ at the Fermi surface. To solve this equation, we expand $v$ and $t$ into Fourier series: $v(\phi)=\sum_{n} v_{n} e^{i n \phi}$, etc. We finally get for the $t$-matrix at the Fermi surface

$$
t(\theta)=\sum_{n} \frac{v_{n}}{1-\nu v_{n}} e^{i n \theta},
$$

with the Fourier coefficients

$$
v_{n}=\frac{4 e_{0}^{2}}{k_{F} \cos \gamma} \sum_{\text {odd } m \geq 1} \frac{\cos (m \gamma)}{2 n+m}
$$

and $\gamma=\arcsin \left(r_{s} / \sqrt{2}\right)$. Below we use this result to evaluate the scattering length.

Scattering length. To illustrate how the scattering process gets renormalized by the Fermi sea, we compare the above result (4) with the $t$-matrix $\left|t_{\mathrm{C}}(\theta)\right|=e_{0} \hbar\left[\tanh \left(\pi m e_{0}^{2} / k_{F} \hbar^{2}\right) \pi / m k_{F} \sin ^{2}(\theta / 2)\right]^{1 / 2}$ obtained for the bare Coulomb potential $V_{C}$ in $2 \mathrm{D}$ [16]. We use typical parameters for a GaAs 2DEG: $\epsilon_{r}=13.1$, $r_{s}=0.86$ and a sheet density $n=4 \cdot 10^{15} \mathrm{~m}^{-2} 19$, and assume $\xi<k_{B} T=10^{-2} E_{F}(T=2 \mathrm{~K})$. In Fig. 2(a) we plot the scattering length $\lambda(\theta)=\left|t m / \hbar^{2} \sqrt{2 \pi p}\right|^{2}$ as function of the scattering angle $\theta$ (without antisymmetrization). The reduction in amplitude due to the Fermi sea is seen to be quite substantial (compared to the bare $t_{C}$ ) which can be traced back to the relatively large screening $k_{s}$ $\left(r_{s}=0.86\right)$. We also have $t \rightarrow 0$ as $k_{\mathrm{B}} T, \xi \rightarrow 0$. For very small $r_{s}$ or increasing $T$ we can drop $\nu v_{n} \ll 1$ in Eq. (4), and thus recover the Born approximation with the bare potential $V_{C}$.

In addition, the higher-order terms appearing in the iteration of the Bethe-Salpeter equation further reduce the scattering (compare $t$ to the first order $V$ ) [20]. In Fig. 2(b) we see the significant reduction of the scattering length $\lambda$ as the density $n$ is increased, which could be tested experimentally.

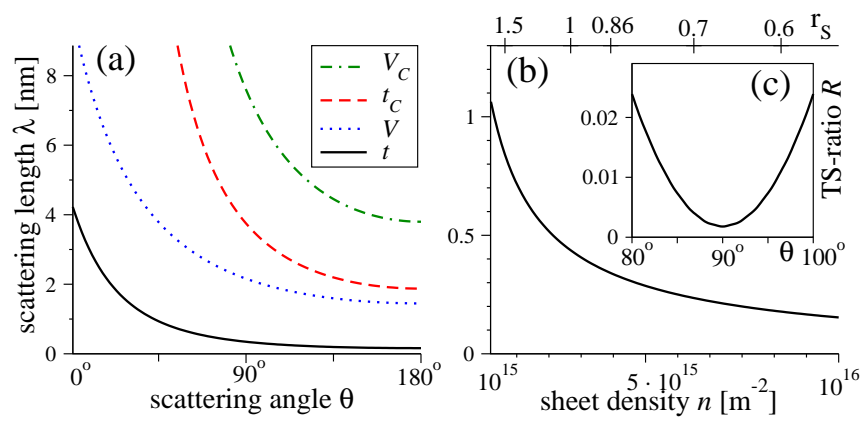

Figure 2: Plots of scattering quantities obtained from the $t$-matrix (4) for $r_{s}=0.86(\mathrm{GaAs})$ and $k_{B} T / E_{F}=10^{-2}$. (a) Angular dependence of the scattering length $\lambda(\theta)$. We compare $\lambda$ to its Born approximation given by $t \simeq V$ and to the bare scattering of two particles (no Fermi sea) given either by the exact result $\left(t_{C}\right)$ or by the first-order $\left(V_{C}\right)$. (b) Dependence of $\lambda(\pi / 2)$ on the sheet density $n$ (see the corresponding $r_{s}=m e_{0}^{2} / \hbar^{2} \sqrt{\pi n}$ in the top axis). (c) TS-ratio $R(\theta, \delta \theta)$ of triplets/singlets detected at a scattering angle $\theta$, for an aperture $\delta \theta=5^{\circ}$.

Production of EPR-pairs. We now turn to the production of entangled electrons in the spin-singlet state. We consider detectors placed at $\theta$, with a small aperture angle of $2 \delta \theta$ (Fig. 1). We introduce the scattering lengths around $\theta$ (integrated over $2 \delta \theta$ ) for singlets $(+)$ and triplets $(-): \bar{\lambda}_{S / T}(\theta)=2 \int_{\theta-\delta \theta}^{\theta} d \theta^{\prime} \mid f\left(\theta^{\prime}\right) \pm f\left(\pi-\left.\theta^{\prime}\right|^{2}\right.$. A useful measure is the ratio between the number $N_{T / S}$ of detected triplets/singlet, $R(\theta, \delta \theta)=N_{T} / N_{S}=3 \bar{\lambda}_{T} / \bar{\lambda}_{S}$. Next, expanding for small $\delta \theta$ around $\theta=\pi / 2$ we find in leading order $\bar{\lambda}_{S} \simeq 8|f(\pi / 2)|^{2} \delta \theta$, and $\bar{\lambda}_{T} \simeq$ $(8 / 3)\left|f^{\prime}(\pi / 2)\right|^{2} \delta \theta^{3}$, which yields

$$
R(\pi / 2, \delta \theta) \simeq\left|\frac{f^{\prime}(\pi / 2)}{f(\pi / 2)}\right|^{2} \delta \theta^{2} .
$$

Using Eq. (4) we find $\left|f^{\prime} / f\right|=0.48$ at $\theta=\pi / 2$ for $\mathrm{GaAs}$, and note that this ratio remains of order unity for a wide parameter range $k_{B} T / E_{F}=10^{-1}-10^{-10}$, and $r_{s}=0.1-1$. The Born approximation $\left|V^{\prime} / V\right|=$ $1 / 2\left(1+r_{s}\right) \simeq 0.266$ is approached for $k_{B} T / E_{F}>10^{-1}$. Therefore, this setup indeed allows the production of dominantly singlets (EPR pairs) at the detectors 1 and 2 provided that the aperture angle is sufficiently small. For example, $R\left(90^{\circ}, 5^{\circ}\right)=0.2 \%$ or $R\left(85^{\circ}, 5^{\circ}\right)=0.7 \%$; see Fig. 2(c).

To estimate the singlet current for a given input current $I$ we assume that the incident electrons occupy the lowest transverse mode in the QPC, giving plane waves of transverse width $w$ (typically $w \simeq 100 \mathrm{~nm}$ ). The probability for the singlets to be scattered into the detectors is $P_{S}=(1 / 4) \bar{\lambda}_{S} / w=0.06 \%$ with $\bar{\lambda}_{S}=0.24 \mathrm{~nm}$ for $\delta \theta=5^{\circ}$. It is advantageous to inject simultaneously the two electrons from the reservoirs (e.g., by opening both QPCs simultaneously) [21]. Then the singlet current is given by $I_{S}=P_{S} I=0.6 \mathrm{pA}$ for $I=1 \mathrm{nA}$. The total scattering length for unpolarized 
electrons is $\lambda_{\text {tot }}=(1 / 4) \int_{-\pi / 2}^{\pi / 2} d \theta\left[\lambda_{S}(\theta)+3 \lambda_{T}(\theta)\right]=3.4$ $\mathrm{nm}$ in GaAs (compared to $11 \mathrm{~nm}$ in Born approximation). This is consistent with the ladder approximation $\left(\lambda_{\text {tot }} k_{F}=0.53\right)$, and yields the total scattering probability $P_{\text {tot }}=\lambda_{\text {tot }} / w=3 \%$.

An important requirement is that only correlated electrons (singlets) are counted at the detectors 1 and 2, i.e. electrons which have scattered off each other at $\theta=\pi / 2$, whereas we need to avoid the counting of uncorrelated electrons which are accidentally scattered into the detectors due e.g. to impurity scattering. This requirement could be fulfilled e.g. by coincidence measurements and/or with the help of an ac modulation applied to each reservoir with different frequencies $\omega_{1}$ and $\omega_{2}$. This would enable a frequency selection of the electrons, since only the electrons which have interacted are modulated by the two frequencies $\omega_{1} \pm \omega_{2}$.

Besides the observation of the scattering length and its density and angular dependence, our proposal could be further tested by adding a beam-splitter to probe the singlet-state via noise measurement [2, 9], by performing tests of Bell's inequality [9, 10] with spin filters 12, 13], or by using p-i-n junctions [22] to transform singlets into entangled photon pairs. An alternative test requires spin-filters, obtained e.g. by tuning the QPCs into the spin-filtering regime [13], or by replacing them by spin-filtering quantum dots [12]. Then with increasing spin polarization $\mathcal{P}$ the probability of incoming singlets $\rho_{S}=\left(1-\mathcal{P}^{2}\right) / 2$ is suppressed, and the singlet current at $\theta=\pi / 2$ vanishes at full polarization $\mathcal{P}=1$.

We now comment on the Kohn-Luttinger instability 14]. The crossed diagram - which in $2 \mathrm{D}$ can lead to an instability only for excitations with $q>2 k_{F}[23]-$ does not lead to a strong renormalization of the scattering vertex, as the associated temperature is infinitesimal, $k_{B} T / E_{F} \sim \exp \left(-10^{3}\right)$ [24]. This is larger than the value $\sim \exp \left(-10^{5}\right)$ found in 3D [14], despite the asymptotic decay for $v_{n} \sim n^{-2}$ being slower than in $3 \mathrm{D}, v_{l} \sim e^{-l}$ (the decay is polynomial because the $2 \mathrm{D}$ potential is nonanalytic). The crossed diagram, given by the polarization propagator, has asymptotics $\sim n^{-3 / 2}$, instead of $\sim l^{-4}$ in 3D. Finally, we also checked that the repulsive electronelectron interaction is not appreciably affected by polar or acoustic phonons [24].

In conclusion, we have shown that the scattering process of two electrons can be used to isolate and select the entangled spin-singlets at the scattering angle $\pi / 2$. The process survives in the presence of an interacting Fermi sea which, however, reduces the scattering amplitude substantially. Nevertheless, according to our analysis the current of entangled electrons is within experimental reach for realistic two-dimensional electron gases.

We thank C. Egues, V. Golovach and W. Coish for useful discussions. This work has been supported by NCCR "Nanoscale Science", Swiss NSF, DARPA and ARO.

[1] Semiconductor Spintronics and Quantum Computation, Eds. D.D. Awschalom, D. Loss, and N. Samarth
(Springer, Berlin, 2002).

[2] G. Burkard, D. Loss, and E.V. Sukhorukov, Phys. Rev. B 61, R16303 (2000).

[3] P. Recher, E.V. Sukhorukov and D. Loss, Phys. Rev. B 63,165314 (2001); G.B. Lesovik, T. Martin, and G. Blatter, Eur. Phys. J. B 24, 287 (2001); P. Recher and D. Loss, Phys. Rev. B 65, 165327 (2002); C. Bena et al., Phys. Rev. Lett. 89, 037901 (2002).

[4] W.D. Oliver, F. Yamaguchi, Y. Yamamoto, Phys. Rev. Lett. 88, 037901 (2002); D.S. Saraga and D. Loss, Phys. Rev. Lett. 90, 166803 (2003).

[5] A.T. Costa, Jr. and S. Bose, Phys. Rev. Lett. 87, 277901 (2001); S. Bose and D. Home, Phys. Rev. Lett. 88, 050401(2002).

[6] P. Samuelsson, E.V. Sukhorukov, and M. Buttiker, cond-mat/0303531 C.W.J. Beenakker et al., cond-mat/0305110

[7] J.R. Taylor, Scattering Theory, Wiley, 1972.

[8] M. A. Topinka et al., Science 289, 2323 (2000); M. A. Topinka et al, Nature 410, 183 (2001).

[9] We emphasize that the detection of spin singlets is a separate issue, discussed e.g. in the review J. C. Egues et al., in Quantum Noise in Mesoscopic Physics, NATO ASI Series II, Vol. 97 (Kluwer, 2003).

[10] S. Kawabata, J. Phys. Soc. Jpn. 70, 1210 (2001).

[11] P.W Anderson, cond-mat/0101417, Phys. Rev. Lett. 71, 1220 (1993) and reference $|1|$ therein.

[12] P. Recher, E.V. Sukhorukov and D. Loss, Phys. Rev. Lett. 85, 1962 (2000).

[13] R.M. Potok et al., Phys. Rev. Lett. 89, 266602 (2002).

[14] W. Kohn and J.M. Luttinger, Phys. Rev. Lett. 15, 524 (1965); J.M. Luttinger, Phys. Rev. 150, 202 (1966).

[15] A.L. Fetter and J.D. Walecka, Quantum Theory of ManyParticle Systems (McGraw-Hill, 1971).

[16] G. Barton, Am. J. Phys. 51, 420 (1983).

[17] We can neglect the $\omega_{q}$ dependence of $\chi^{0}$ as it is a smooth function and the sharp Fermi surface selects $\xi_{k}=0 \Rightarrow \hbar \omega_{q}=\xi_{k}-\xi_{1}=0$ for $\xi_{1} \rightarrow 0$. Note that the $\omega_{q}$-integration of $V(\tilde{q}) \sim 1 /\left(\omega_{q}-\omega_{p}\right)$ near the divergence at the plasmon frequency $\omega_{p}$ vanishes as it is an odd function of $\omega$. Finally, the $q$-dependent part of $\chi^{0}$ vanishes in $2 \mathrm{D}$ for $q \simeq 2 k_{F}|\sin \theta / 2|<2 k_{F}$; see F. Stern, Phys. Rev. Lett. 18, 546 (1967).

[18] A.V. Chubukov and M. Yu Kagan, J. Phys.: Condens. Matter 1, 3135 (1989).

[19] T. Ando, A.B. Fowler, and F. Stern, Rev.Mod.Phys 54, 437 (1982).

[20] Outside the Cooper channel, in the absence of the logarithmic divergence (3), the Born approximation is valid.

[21] For random injection times, the singlet current would scale down with the probability occupation for $\left|\mathbf{p}_{1} \mathbf{p}_{2}\right\rangle$, given roughly by $P_{12} \sim\left(e W_{\text {out }} / I\right)^{2}$, with $W_{\text {out }}$ the rate for electrons to escape out of the scattering region.

[22] R. Fiederling et al., Nature 402, 787 (1999); Y. Ohno et al., Nature 402, 790 (1999).

[23] It is necessary to go beyond second order in the irreducible vertex to find an instability also for $q<2 k_{F}$; see A. V. Chubukov, Phys. Rev. B 48, 1097 (1993).

[24] D.S. Saraga et al., unpublished. 